\title{
LV Motion Tracking from 3D Echocardiography Using Textural and Structural Information
}

\author{
Andriy Myronenko ${ }^{1}$, Xubo Song ${ }^{1}$, and David J. Sahn ${ }^{2}$ \\ ${ }^{1}$ Dept. of CSEE, OGI School of Science and Engineering \\ ${ }^{2}$ Cardiac Fluid Dynamics and Imaging Laboratory \\ ${ }^{1,2}$ Oregon Health and Science University \\ 20000 NW Walker Road, Beaverton, OR 97006, USA \\ myron@csee.ogi.edu, xubosong@csee.ogi.edu, sahnd@ohsu.edu
}

\begin{abstract}
Automated motion reconstruction of the left ventricle (LV) from 3D echocardiography provides insight into myocardium architecture and function. Low image quality and artifacts make 3D ultrasound image processing a challenging problem. We introduce a LV tracking method, which combines textural and structural information to overcome the image quality limitations. Our method automatically reconstructs the motion of the LV contour (endocardium and epicardium) from a sequence of $3 \mathrm{D}$ ultrasound images.
\end{abstract}

\section{Introduction}

The analysis of myocardium deformation provides insight into the heart's architecture and function. Medical imaging techniques, such as MRI, CT and recently 3D ultrasound, allow the acquisition of dynamic sequences of 3D images $(3 \mathrm{D}+\mathrm{T})$ during a complete cardiac cycle. Processing these images can provide quantitative measurements such as strain, wall thickening, torsion, volume and ejection fraction, which can be used to evaluate the elasticity and contractility of the myocardium. For instance, an ischemic or infarcted segments of the heart are typically associated with reduced regional elasticity and contractility. Such measurements may also serve as earlier sub-clinical markers for ventricular dysfunction and myocardial disease [1/2].

3D echocardiography provides an attractive alternative to MRI and CT because of its portability, bedside applicability, low cost, and safety benefits [1. Despite certain advances in technology, 3D ultrasound images are still of low quality with many artifacts, such as attenuation, speckle, shadows, and signal dropout. A relatively small amount of research has been done in motion analysis from 3D echocardiography [345]. Montagnat and colleagues [4] develop a 3D model-based ultrasound segmentation; the method filters the image by (4D) anisotropic diffusion and fits the LV model to the high intensity gradients. Sanchez-Ortiz and collaborators $[5$ use multi-scale fuzzy-clustering method together with phase-based acoustic feature detection to fit the LV model. We call such methods, which incorporate a model-based boundary segmentation, structure based methods.

N. Ayache, S. Ourselin, A. Maeder (Eds.): MICCAI 2007, Part II, LNCS 4792, pp. 428-435, 2007.

(C) Springer-Verlag Berlin Heidelberg 2007 
Low image contrast of 3D echocardiography makes the LV boundaries challenging for segmentation. Instead of tracking the LV boundaries, speckle tracking was proposed to analyze myocardium deformation 6 6]. Speckle is a texture pattern formed by the interference of the backscattered echoes produced by ultrasonic scatterers in tissue and blood. The speckles follow the motion of the myocardium and remain fairly constant when the temporal sampling rate is adequately high, which makes tracking possible. We call such methods, which incorporate ultrasound beam amplitude/phase, postprocessed intensity or its transformations, texture based methods.

We present a method for tracking the LV motion, given a time sequence of 3D ultrasound images. We use two sources of information for tracking: textural and structural. We use textural information (e.g. speckle intensity) to align two consecutive images. We use structural information to assure that alignment using textural information locates the LV contour at the heart boundaries. Thus, we seek the position of the LV contour in the next time volume, so that its texture coinsides with the texture of its previous position, and so that its location is on the heart's boundary. The implementation of our method has a low computation complexity, which is desirable in clinical settings. Combining textural and structural information can greatly improve the accuracy of the motion tracking in $3 \mathrm{D}$ ultrasound imaging.

\section{Combining Textural and Structural Information}

Consider two 3D ultrasound images, $I_{t}(x, y, z)$ and $I_{t+1}\left(x^{\prime}, y^{\prime}, z^{\prime}\right)$, obtained at time $t$ and $t+1$ respectively. Assume an initial boundary segmentations of the LV endocardium and epicardium are available at time $t$. These two boundaries define the LV contour $C_{t}$. Boundary initialization is itself a complicated problem; we will describe a procedure in Section 3.4. For tracking, our goal is to find the contour position $C_{t+1}$ at time $t+1$.

Using the textural information, we register image $I_{t}$ with $I_{t+1}$ and use the resulting transformation to transform the initial contour $C_{t}$ to its new position $C_{t+1}$. The goal of image registration is to find the underlying transformation $T:(x, y, z) \rightarrow\left(x^{\prime}, y^{\prime}, z^{\prime}\right)$ that maps any point of one image into its corresponding point in the other image. In our implementation, we use image intensity as a textural representation of the images; other features, such as image gradient or moments, are also appropriate.

Using the structural information, we want to align the contour $C_{t}$ to the high intensity gradient region of the image $I_{t+1}$. We adopt the active contour segmentation approach [8], while using the same FFD transformation $T\left(C_{t+1}=\right.$ $\left.T\left(C_{t}\right)\right)$ as for the image registration.

The motion of left ventricle is complex. We choose a Free Form Deformation (FFD) model 9] to parameterize the transformation T. FFD has been successfully used for non-rigid medical image registration [10] and for tracking of cardiac MRI 11. The basic idea of FFD is to deform a 3D object by manipulating a mesh of control points. Since FFD is volumetric transformation, we can transform 

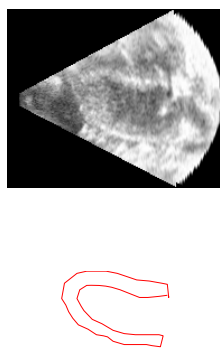

(a)

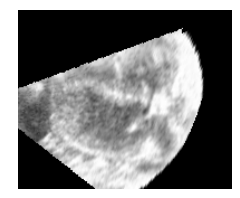

?

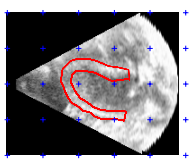

$T$
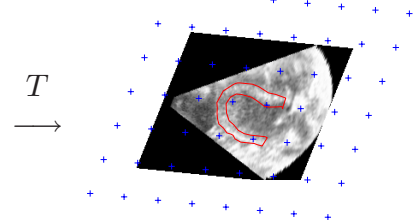

(b)

Fig. 1. Schema of the method. (a) Given two 3D ultrasound images $I_{t}$ and $I_{t+1}$, and the initial position of the contour $C_{t}$, find the contour position $C_{t+1}$. (b) We use FFD to parametrize the transformation $T$ by a mesh of control points $(+)$. By manipulating the control points positions, we want to solve two tasks simultaneously: to deform the image $I_{t}$ to align it with $I_{t+1}$, and to deform contour $C_{t}$ to align it to the high intensity gradient region of the image $I_{t+1}$.

several objects (e.g., 3D image, endocardium and epicardium contours) simultaneously with a single FFD. The main advantage of FFD is that the complex non-rigid transformation is defined by a small number of parameters (control points positions).

Our tracking method is a joint non-rigid image registration and active contour segmentation, where we use a single FFD parametrization of transformation $T$. We minimize the energy function

$$
E=E_{\text {text }}+\lambda E_{\text {struct }}
$$

where $E_{\text {text }}$ is a non-rigid image registration objective function that represents a texture based tracking, and $E_{\text {struct }}$ is an active contour energy function that represents a structure based tracking. Parameter $\lambda$ represents a trade-off between the textural and structural information influences. Figure 1illustrates the schema of the method.

\section{Method}

\subsection{Non-rigid Image Registration Using FFD}

We use FFD framework 910] to parametrize the non-rigid transformation $T$. We denote the image volume as $\Omega=\left\{(x, y, z) \mid 0 \leq x_{n} \leq N, 0 \leq y_{m} \leq M, 0 \leq\right.$ $\left.z_{k} \leq K\right\}$. We place a $n_{x} \times n_{y} \times n_{z}$ mesh of equally spaced control points $p_{i, j, l}$ over the image domain. The number of control points defines the complexity of the transformation. Then, the transformation $T$ is a $3 \mathrm{D}$ tensor product of the 1D cubic B-splines:

$$
\left(x^{\prime}, y^{\prime}, z^{\prime}\right)=T(x, y, z ; p)=\sum_{k=0}^{3} \sum_{m=0}^{3} \sum_{n=0}^{3} B_{k}\left(u_{x}\right) B_{m}\left(v_{y}\right) B_{n}\left(w_{z}\right) p_{i+k, j+m, l+n}
$$


where $i=\left\lfloor x / n_{x}\right\rfloor-1, j=\left\lfloor y / n_{y}\right\rfloor-1, l=\left\lfloor z / n_{z}\right\rfloor-1, u_{x}=x / n_{x}-\left\lfloor x / n_{x}\right\rfloor, v_{y}=$ $y / n_{y}-\left\lfloor y / n_{y}\right\rfloor, w_{z}=z / n_{z}-\left\lfloor z / n_{z}\right\rfloor$, and $B_{k}$ represents the $k^{\text {th }}$ B-spline basis function:

$$
\begin{array}{ll}
B_{1}(u)=(1-u)^{3} / 6 & B_{2}(u)=\left(3 u^{3}-6 u^{2}+4\right) / 6 \\
B_{3}(u)=\left(-3 u^{3}+3 u^{2}+3 u+1\right) / 6 & B_{4}(u)=u^{3} / 6
\end{array}
$$

Putting all control points in a matrix $\mathbf{P}_{n_{x} n_{y} n_{z} \times 3}$, we can rewrite Eq. 2 as

$$
\mathbf{X}^{\prime}=T(\mathbf{X}, \mathbf{P})=\mathbf{B P}
$$

where $B_{M N K \times n_{x} n_{y} n_{z}}$ is a sparse matrix of basis function values with $M N K \times 64$ non-zero elements. We precompute the matrix $\mathbf{B}$ taking advantage of the known $3 \mathrm{D}$ grid structure of digital images. Matrix $\mathbf{X}_{M N K \times 3}$ denotes the coordinates of all voxels in the image $I_{t}$. We want to align image $I_{t}$ with $I_{t+1}$ by minimize the following energy function:

$$
\begin{aligned}
E_{\text {text }}(\mathbf{P})= & \sum_{n, m, k=0}^{N, M, K}\left(I_{t+1}\left(T\left(x_{n}, y_{m}, z_{k}, \mathbf{P}\right)\right)-I_{t}\left(x_{n}, y_{m}, z_{k}\right)\right)^{2}= \\
& \sum\left(I_{t+1}(T(\mathbf{X}, \mathbf{P}))-I_{t}(\mathbf{X})\right)^{2}=\sum\left(I_{t+1}(\mathbf{B P})-I_{t}\right)^{2}
\end{aligned}
$$

where $I_{t+1}(\mathbf{B P})$ represents a column vector of image intensities at coordinates $\mathbf{X}^{\prime}=\mathbf{B P}$, and summation is over all image voxels.

Little attention has been paid to image registration for 3D echocardiography motion analysis 12. To our best knowledge, this paper is the first that applies FFD for 3D echocardiography non-rigid registration and motion tracking.

\subsection{Active Contour Segmentation}

We use structural information to assist the image registration process by applying the active contour approach [8] to align the contour $C_{t}$ to the high intensity gradient region of the image $I_{t+1}$. We use the same transformation $T$ as for the image registration to parametrize the contour deformation: $C_{t+1}=T\left(C_{t}, \mathbf{P}\right)$.

The active contour moves to minimize its energy function, which consist of internal and external energy. The internal energy keeps the active contour smooth; the FFD parametrization of $T$ innately constrains the contour motion by construction [1]. The external energy has lower intensity values at the desirable position of the active contour. For the external energy, we use a negative gradient norm of 3D ultrasound images preprocessed by 3D anisotropic diffusion [13. This way we remove the texture information, preserve the contrast, and emphasize the boundaries 4. To reduce the computational complexity, we discretize the initial contour $C_{t}$ and precompute the B-splines basis function values in the matrix $\mathbf{D}$. We minimize the energy function:

$$
\mathbf{E}_{\text {struct }}(\mathbf{P})=-\sum F_{t+1}\left(C_{t+1}\right)=-\sum F_{t+1}\left(T\left(C_{t}, \mathbf{P}\right)\right)=-\sum F_{t+1}(\mathbf{D P})
$$

where $F_{t+1}$ denotes the feature-map of the image $I_{t+1}$. 


\section{LV contour tracking}

- Initialize the contour $C_{1}$ in the first volume (Sec. 3.4)

- Uniformly allocate the mesh of B-splines control points $\mathbf{P}$ over the image domain

- Precompute B-splines matrices B and D.

- For $t=2: T$ (through all volumes):

- Minimize the objective function (Eq. 6) with respect to control points positions $\mathbf{P}_{t}$, using the steepest descent method (Sec. 3.3).

- New contour position is $C_{t}=\mathbf{D P}_{t}$

Fig. 2. Pseudo-code of LV contour tracking in 3D ultrasound image sequence

\subsection{Optimization}

To minimize the energy function in Eq. 1, we rewrite it as

$$
E(\mathbf{P})=\sum\left(I_{t+1}(\mathbf{B P})-I_{t}\right)^{2}-\lambda \sum F_{t+1}(\mathbf{D P})
$$

Taking the gradient of the function with respect to $\mathbf{P}$, we obtain

$$
\frac{\partial E(\mathbf{P})}{\partial \mathbf{P}}=2 \mathbf{B}^{T}\left[I_{t+1}(T(\mathbf{X}, \mathbf{P}))-I_{t}(\mathbf{X})\right] \nabla I_{t+1}-\lambda \mathbf{D}^{T} \nabla F_{t+1}(T(\mathbf{X}, \mathbf{P}))
$$

We use a steepest descent optimization method to minimize the energy function.

\subsection{Contour Initialization}

Our approach requires an initial contour segmentation $C_{t}$. We choose an approach similar to Bardinet et al. 11 to parametrize the LV contour. We start from two parametric half-ellipsoids, parametrized by 9 parameters: 6 parameters for rigid transformation and 3 for scaling. We use the active contour approach, similar to Sec. 3.2, to roughly prealign two half-ellipsoids to the endocardium and epicardium. Then we fix the rigid motion and scaling parameters, parametrize both half-ellipsoids by a single FFD, and refine the segmentation. The FFD, rigid and scaling parameters define the parametric form of the initial contour $C_{t}$. The contour is parametric, because parametric objects remain parametric after FFD. The FFD used for initial segmentation is not related to the FFD parametrization used in our tracking method. Once we obtain the initial segmentation $C_{t}$, all its parameters remain constant during tracking. We use another set of B-spline control points to parametrize transformation $T$. Furthermore, we discretize $C_{t}$ to reduce computational complexity by precomputing the corresponding B-spline basis function values in the matrix $D$. We summarize our LV tracking method in Fig. 2 .

\section{Results}

We test the method on a set of $3 \mathrm{D}$ ultrasound sequences $(3 \mathrm{D}+\mathrm{T})$ from openchest porcine hearts. The scans are acquired using Philips Sono 7500 with EKG 
Half-ellipsoids. FFD refinement. Initial countour segmentation $\left(C_{t}\right)$.
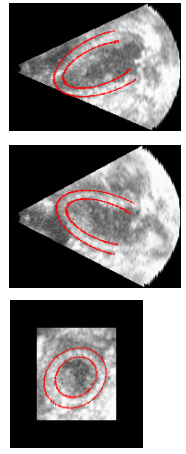
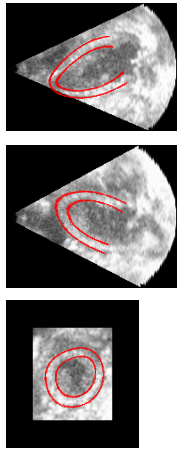

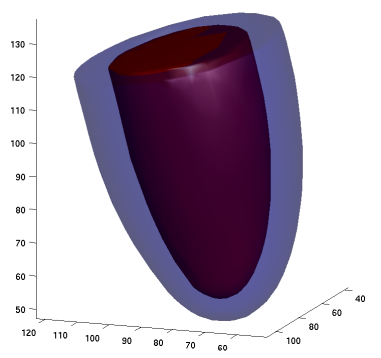

Fig. 3. Boundaries initialization procedure. The first column shows the segmentation results using two half-ellipsoids. Three perpendicular 2D cross planes are demonstrated. The second column shows the FFD refinement of the segmentation. The last image shows the initial contour $C_{t}$.

gating. We use detected post-scan converted images with echo envelop signals, represented in the Cartesian coordinates. The spatial resolution is $160 \times 144 \times 192$ voxels. Ten different scans $(3 \mathrm{D}+\mathrm{T})$ are taken from a single pig. Each scan consists of ten $3 \mathrm{D}$ volumes. We run the experiments for each of the scans.

We implement the algorithm in Matlab, and test it on a Pentium4 CPU $3 \mathrm{GHz}$ with 4GB RAM. For the anisotropic diffusion parameters, we use exponential function with $K=0.2$ and limit the number of iterations to 20 [13. We put the FFD control points uniformly with 10 voxels spacing, which makes it a $16 \times 15 \times 20$ mesh. We use the trade-off parameter $\lambda=0.1$, which we found empirically to give the best performance. On average, the tracking algorithm between two volumes converges in less than 10 minutes and requires around 150 iterations.

We show the initialization procedure for a particular scan in Fig. 3. First, we use the active contour approach described in Sec. 3.4 to segment the images using 2 half-ellipsoids parametrized by the rigid motion. Second, we use FFD to refine the contour segmentation $C_{t}$. Having the initial contour, we track the LV position through the sequence. We show the recovered LV motion in Fig. 4.

For validation, we define 12 points on endocardium and epicardium and manually track them across the sequence. We ask three interpreters to track the points manually, given its initial locations. We provide them with a tool to visualize and select the points for the arbitrary 2D cross section of $3 \mathrm{D}$ volume. The cross-expert variation is 0.6315 voxels, averaged over time. We use mean values of obtained points' trajectories as a ground truth of the points motion. We compare our automated tracking against ground truth: The tracking error (averaged over all scans, volumes and number of speckles) is $1.0311 \pm 0.6265$ voxel. 


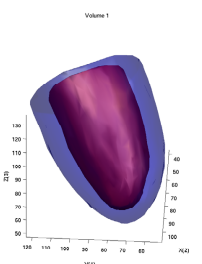

volume 1

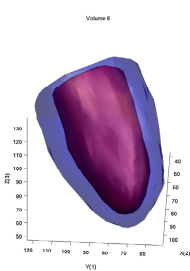

volume 6

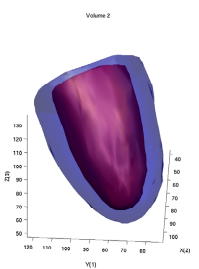

volume 2

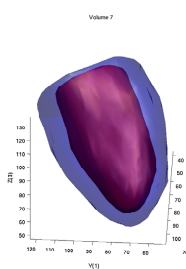

volume 7

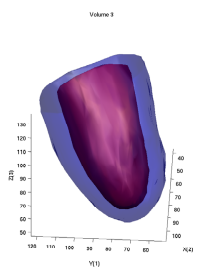

volume 3

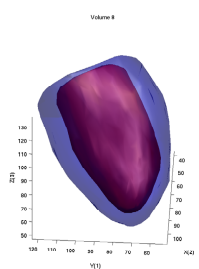

volume 8

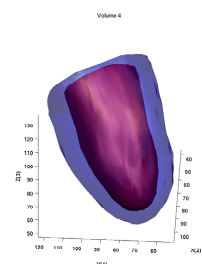

volume 4

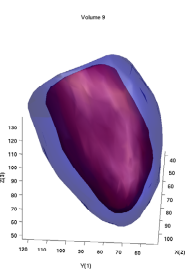

volume 9

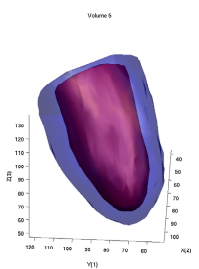

volume 5

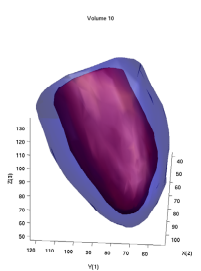

volume 10

Fig. 4. The contour position found during the cardiac cycle (10 consecutive volumes). LV achieves the maximum contraction at volume 3, then the LV dilates (diastolic phase) up to the volume 9 and starts contracting again (systolic phase).

Table 1. Mean-square-error (MSE) of tracking results using combined objective function, only textural, only structural or without registration

\begin{tabular}{l|l} 
Objective function & MSE (voxels) \\
\hline$E_{\text {text }}+\lambda E_{\text {struct }}$ & $1.0311 \pm 0.6265$ \\
$E_{\text {text }}$ & $3.2141 \pm 1.3442$ \\
$E_{\text {struct }}$ & $10.2571 \pm 4.5941$ \\
without registration & $17.2331 \pm 5.1224$
\end{tabular}

We also consider two extreme cases when $\lambda=0$ or $\lambda=$ inf, which correspond to textural information only or structural information only cases. For instance, with $\lambda=0$, we optimize only the $E_{\text {text }}$ objective function. We register all images and use the transformation to propagate the motion of the initial contour $C_{t}$. With $\lambda=$ inf, we consequently segment the images starting form the initial contour $C_{t}$. We show the mean square error (MSE) of tracking results using only textural, only structural, both objective functions and without any registration (initial positions remain constant) in Table 1. Our experiments show that accurate motion tracking in 3D ultrasound benefits from using both textural and structural information.

\section{Discussion and Conclusions}

We present a method for LV motion tracking in 3D ultrasound. The method combines textural and structural information to overcome the low 3D ultrasound image quality. We use non-rigid image registration, parametrized by FFD, 
to take advantage of textural information. We also use model-based active contour segmentation to take advantage of structural information. We show the optimization algorithm for the joint non-rigid registration and segmentation.

Our non-rigid image registration implementation uses a sum of squared differences similarity function, which implies a Gaussian noise assumption between the images. Other similarity functions, such as. those based on the Rayleigh multiplicative noise model, can also be used within this framework. We use 3D ultrasound B-mode images. The alternative of using radio frequency data may provide extra information in the axial direction, but the increased computation load may be prohibitive for real applications.

Our method has a low computational complexity due to the FFD parametrization with precomputed basis function values for registration and segmentation. We demonstrate the performance of the method on 10 different 3D echocardiography scans from open chest porcine hearts; the method shows accurate and robust performance.

\section{References}

1. Lang, M., Mor-Avi, V., Sugeng, L., Nieman, P., Sahn, J.: Three-dimensional echocardiography. Jour. of the Am. Coll. of Cardiology 48(10), 2053-2069 (2006)

2. Papademetris, X., Sinusas, A.J., Dione, D.P., Constable, R.T., Duncan, J.S.: Estimation of 3D left ventricular deformation from medical images using biomechanical models. IEEE Trans. Med. Imaging 21(7), 786-800 (2002)

3. Noble, A.J., Boukerroui, D.: Ultrasound image segmentation: a survey. IEEE Transactions on medical imaging 25(8), 987-1010 (2006)

4. Montagnat, J., Sermesant, M., Delingette, H., Malandain, G., Ayache, N.: Anisotropic filtering for model-based segmentation of $4 \mathrm{D}$ cylindrical echocardiographic images. PRL- S. Issue on Ultr. Im. Proc. and Anal. 24(4-5), 815-828 (2003)

5. Sanchez-Ortiz, G.I., Declerck, J., Mulet-Parada, M., Noble, J.A.: Automating 3D echocardiographic image analysis. In: Delp, S.L., DiGoia, A.M., Jaramaz, B. (eds.) MICCAI 2000. LNCS, vol. 1935, pp. 687-696. Springer, Heidelberg (2000)

6. Chen, X., Xie, H., Erkamp, R., Kim, K., Jia, C., Rubin, J.M., O’Donnell, M.: 3-D correlation-based speckle tracking. Ultrasonic Imaging 27, 21-36 (2005)

7. Song, X., Myronenko, A., Sahn, D.J.: Speckle tracking in 3D echocardiography with motion coherence. In: Comp. Vision and Patt. Recognition (CVPR) (2007)

8. Kass, M., Witkin, A., Terzopoulos, D.: Snakes: Active contour models. Inter. J. Computer Vision 1(4), 321-331 (1988)

9. Sederberg, T.W., Parry, S.R.: Free-form deformation of solid geometric models. In: SIGGRAPH, pp. 151-160 (1986)

10. Rueckert, D., Sonoda, L.I., Hayes, C., Hill, D.L.G., Leach, M.O., Hawkes, D.J.: Nonrigid registration using free-form deformations: Application to breast MR images. IEEE Trans. Image Processing 18(8), 712-721 (1999)

11. Bardinet, E., Cohen, L., Ayache, N.: Tracking and motion analysis of the left ventricle with deformable superquadrics. Medical Image Analysis 1(2) (1996)

12. Makela, T., Clarysse, P., Sipila, O., Pauna, N., Pham, Q.C., Katila, T., Magnin, I.: A review of cardiac im. reg. meth. IEEE Tr. on Med.Im. 21(9), 1011-1021 (2002)

13. Perona, P., Malik, J.: Scale-space and edge detection using anisotropic diffusion. IEEE Trans. Pattern Anal. Machine Intell. 12(7), 629-639 (1990) 